\title{
Clinical and Economic Characteristics of Milestones along the Continuum of Alzheimer's Disease: Transforming Functional Scores into Levels of Dependence
}

\author{
K. Kahle-Wrobleski1, J.S. Andrews' 1 , M. Belger2, S. Gauthier ${ }^{3}$, Y. Stern ${ }^{4}$, D.M. Rentz 5 , D. Galasko 6
}

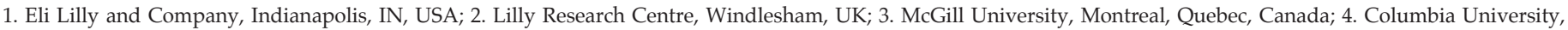
New York, NY, USA; 5. Harvard Medical School, Boston, MA, USA; 6. University of California San Diego, San Diego, CA, USA

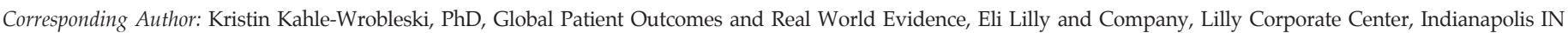
46285, USA, Tel: 317-651-9881; Fax: 317-276-5791; Email: wrobleskikr@lilly.com

J Prev Alz Dis 2015;2(2):115-120

Published online January 20, 2015, http:/ / dx.doi.org/10.14283/jpad.2015.53

\begin{abstract}
BACKGROUND: Because Alzheimer's disease (AD) is characterized by a gradual decline, it can be difficult to identify distinct clinical milestones that signal disease advancement. Adapting a functional scale may be a useful way of staging disease progression that is more informative for healthcare systems.

OBJECTIVES: To adapt functional scale scores into discrete levels of dependence as a way of staging disease progression that is more informative to care providers and stakeholders who rely on the functional impact of diseases to determine access to supportive services and interventions.

DESIGN: Analysis of data from the GERAS study.

SETTING: GERAS is an 18-month prospective, multicenter, naturalistic, observational cohort study reflecting the routine care of patients with AD in France, Germany, and the United Kingdom.

PARTICIPANTS: Data were from baseline results of 1497 community-living patients, aged $\geq 55$ years, diagnosed with probable AD and their caregivers.

MEASUREMENTS: We used data from the Alzheimer's Disease Cooperative Study Activities of Daily Living Inventory (ADCS-ADL) and mapped items onto established categories of functional dependence, validated using clinical and economic measures. Cognitive function, behavioral symptoms, caregiver burden, and cost were assessed. Based on stages of functional dependence described by the Dependence Scale, individual ADCS-ADL items were used to approximate 6 dependence levels.

RESULTS: There was a significant relationship between assigned level of dependence derived from the ADCS-ADL score and cognitive severity category. As the assigned level of dependence increased, the associated clinical and economic indicators demonstrated a pattern of greater disease severity. CONCLUSIONS: This mapping provides initial support for dependence levels as appropriate interim clinical milestones that characterize the functional deficits associated with AD.
\end{abstract}

Key words: Alzheimer's disease, observational study, informal care, caregiver burden.

\section{Introduction}

lzheimer's disease (AD) is a chronic,
progressive illness characterized by a decline
in memory and cognitive abilities, loss of capacity in activities of daily living (ADL), reduced global functioning, and behavioral and psychological symptoms. Because the disease is characterized by a gradual decline, it can be difficult to identify clear milestones that signal clinically meaningful disease advancement. Transforming functional scale scores into discrete levels of disability may be a useful way of staging disease progression that is more informative to a variety of care providers and stakeholders who rely on the functional impact of diseases to determine access to supportive services and interventions. Furthermore, most previous studies of disease advancement have focused on one facet such as cognition or functional ability. Many different functional scales are used in clinical trials and observational studies of patients with $\mathrm{AD}$, so finding a common heuristic across different scales would be useful to facilitate comparisons and build common understandings.

Zhu et al. (2009) (1) recognized that deficits associated with $\mathrm{AD}$, including deficits in cognition, function, and behavior, could be assessed with a single scale. They used the Dependence Scale (DS) (2) to assess the overall impact of these impairments because increasing impairments would lead to greater dependence on others (e.g., caregivers) for required care and service needs. They found that patient dependence was associated with the 3 clinical endpoints as well as measures of cost. Jones et al. (2014) (3) proposed that dependence could be a marker for following disease progression. They found the DS to be a useful tool for assessing patients with AD because it combines the impact of changes in cognition, function, and behavior. They also found significant associations between scores on the DS and cost, patient quality of life, and caregiver perceived burden.

With the objective of finding a common heuristic 
across different scales, we mapped the Alzheimer's Disease Cooperative Study Activities of Daily Living Inventory (ADCS-ADL) items onto levels of dependence derived from the DS definitions. This mapping was validated using additional clinical and economic measures.

\section{Methods}

\section{Participants and Study Design}

GERAS is an 18-month, prospective, multicenter, naturalistic, observational, cohort study reflecting the routine care of patients with AD in France, Germany, and the United Kingdom, with a further 18-month followup period in France and Germany. The study design and methods have been described elsewhere (4). Briefly, investigators enrolled community-living patients, aged $\geq 55$ years, diagnosed with probable $\mathrm{AD}$ according to the National Institute of Neurological and Communicative Disorders, and Stroke and Alzheimer's Disease and Related Disorders Association criteria (NINCDSADRDA) (5), with a Mini-Mental State Examination (MMSE) (6) score of $\leq 26$, who were evaluated within the normal course of care. A further inclusion criterion was that the patient's primary caregiver (other than a healthcare professional) was willing to participate in the study. To be included in the study, the caregiver had to be responsible for the patient for at least 6 months of the year. Patients with other potential causes of dementia were excluded from the study. All patients (or their legal representatives) and caregivers provided written informed consent and the study was approved by ethical review boards in each country.

\section{Patient and Caregiver Data}

Baseline data were evaluated for 1497 patients and their caregivers. Functional ability was assessed using the Alzheimer's Disease Cooperative Study Activities of Daily Living Inventory (ADCS-ADL), a caregiverreported measure of a patient's dependence in basic and instrumental (complex) ADL. Cognitive function was tested using the MMSE. Behavioral symptoms were assessed using the 12-item Neuropsychiatric Inventory
(NPI) (7). Direct and indirect resource use was assessed using the Research Utilization in Dementia Scale (RUD) (8). Country-specific costs were assigned to each unit of resource utilization from the RUD. Information regarding patient health status was collected from caregivers via a proxy version of the EuroQol-5 Dimension (EQ-5D) (9). Caregivers also completed the Zarit Burden Inventory (ZBI) (10), a measure of caregivers' subjective burden related to caring for a loved one.

For the analysis, patients were stratified according to disease severity at baseline using MMSE criteria: mild AD (MMSE 21-26 points), moderate AD (MMSE 15-20 points), or moderately severe/severe AD (MMSE <15) (based in part on NICE Guidance 217) (11).

\section{Categorizing Dependence Levels}

The DS includes a scheme to derive a dependence level based on responses to the individual items. Based on the DS items included in each of these dependence levels, a theoretical scheme was devised for defining stages of functional dependence (Table 1). The pattern of responses depicting type and degree of impairment on individual items from the ADCS-ADL were used to approximate the 6 dependence levels, ranging from no instrumental ADL or basic ADL impairment (Level 0) to impaired transfer or complete incontinence (Level 5). Mapping from the ADCS-ADL questionnaire to the functional levels is shown in Table 2.

Confirmatory factor analysis of ADCS-ADL data was conducted in order to create subscales to aid in the construction of dependence levels (12). Baseline data suggested a 4-factor solution that included factors for BADLs, domestic/household activities, communication/ engagement with the environment, and outside activities (Figure 1).

Once categorizations were completed, the profile of patients in each category was determined using the following measures: MMSE, EQ-5D (proxy), NPI, ZBI, total caregiver time, total societal costs, and patient medical costs. Mean values and $95 \%$ confidence intervals were calculated for scales in each functional dependence category.

\begin{tabular}{|c|c|c|}
\hline Level & Types of Impairments & Care Needs \\
\hline 0 & No IADL/BADL impairment & None \\
\hline 2 & $\begin{array}{l}\text { Supervision on multiple IADLs or loss of at least } 1 \\
\text { Household Activity }\end{array}$ & Limited/informal home care services \\
\hline 4 & Supervision on some BADLs & Assisted living + nursing support \\
\hline 5 & Impaired transfer OR complete incontinence & Nursing home \\
\hline
\end{tabular}

Abbreviations: BADL, Basic Activities of Daily Living; IADL, Instrumental Activities of Daily Living 
Table 2. Mapping from the ADCS-ADL questionnaire to the functional dependence levels

\begin{tabular}{|l|l|}
\hline Level & Scoring Algorithm versus Care Needs \\
\hline 1 & No impairments in ADCS-ADL \\
2 & $\begin{array}{l}\text { Level } 2 \text { on any } 1 \text { item from the following clusters: Household activities, Communication and Engagement, Outside } \\
\text { Activities }\end{array}$ \\
& $\begin{array}{l}\text { Level } 2 \text { on items from at least } 2 \text { of the following clusters: Household activities, Communication and Engagement, } \\
\text { Outside Activities, OR Level } 1 \text { on any item from the following clusters: Household activities, Communications and } \\
\text { Engagement, Outside Activities, } \\
\text { OR Level } 0 \text { on any } 1 \text { item of Household activities }\end{array}$ \\
3 & $\begin{array}{l}\text { Level } 2 \text { for all items from the following clusters: Household activities, Communications and Engagement, Outside } \\
\text { Activities, OR Level } 0 \text { on } 1 \text { item of Outside Activities, OR Level } 2 \text { for either item: Eating (Q1), Bathing (Q4) }\end{array}$ \\
4 & $\begin{array}{l}\text { Score }<2 \text { for item Dressing (Q6B), OR Level } 1 \text { or } 0 \text { or for any items: Grooming (Q5), Bathing (Q4), OR Level 2 for item } \\
\text { Toileting (Q3), OR Level } 0 \text { for Eating (Q1) }\end{array}$ \\
5 & Level 1 for Walking (Q2), OR Level 0 for Toileting (Q3)
\end{tabular}

Abbreviations: ADCS-ADL, Alzheimer's Disease Cooperative Study-Activities of Daily Living; Q, question; Note: Q16B (Outdoor activity question) is not included); Q20 (Communication question) only has a maximum level of 2 , so the question is one point lower in the above settings.

\section{Statistical Analysis}

All patients and associated caregivers who provided informed consent and fulfilled the study entry criteria were included in the present analysis.

Descriptive statistics (means and standard deviations [SDs] or frequencies) were used to summarize all variables and were based on non-missing observations. Comparisons between AD severity groups used Cochran-Mantel-Haenszel tests for categorical data, stratified by country; for continuous data, analysis of variance (ANOVA) was used with independent factors for MMSE severity and country.

The relationship between dependence levels and cognitive severity was assessed using the CochranMantel-Haenszel test. Generalized linear models (GLM) were run to test for differences between ADL levels for all outcome measures. For caregiver time, total societal costs and patient medical costs a GLM was fitted with a gamma distribution and log-link function, all other outcomes used GLM's with a normal distribution and an identity link function.

All data were analyzed using SAS software, version 9.2 (SAS Institute, Cary, North Carolina, USA).

\section{Results}

Overall, 1532 patients and their primary caregivers were enrolled by 94 investigators. After excluding 35 patients who did not fulfill the study entry criteria, a total of 1497 patients and caregivers were included in the analyses.

\section{Descriptive Statistics}

A summary of the patient characteristics is shown in Table 3. Full baseline patient characteristics were previously reported (4). The patients' mean age was 77.6 years and most patients were female (54.8). The mean MMSE score was 17.4.

The caregivers' mean age was 67.3 years. Most of the caregivers were female $(64.1 \%)$ and $65.9 \%$ were spouses of the patients. The mean ZBI score was 29.0.

There was a significant relationship between the assigned level of dependence derived from the ADCSADL score (0 to 5 ) and the cognitive severity category (mild/moderate/moderately severe or severe). Severity level increased with level of dependence $(\mathrm{p}<0.001$; Figure 2).

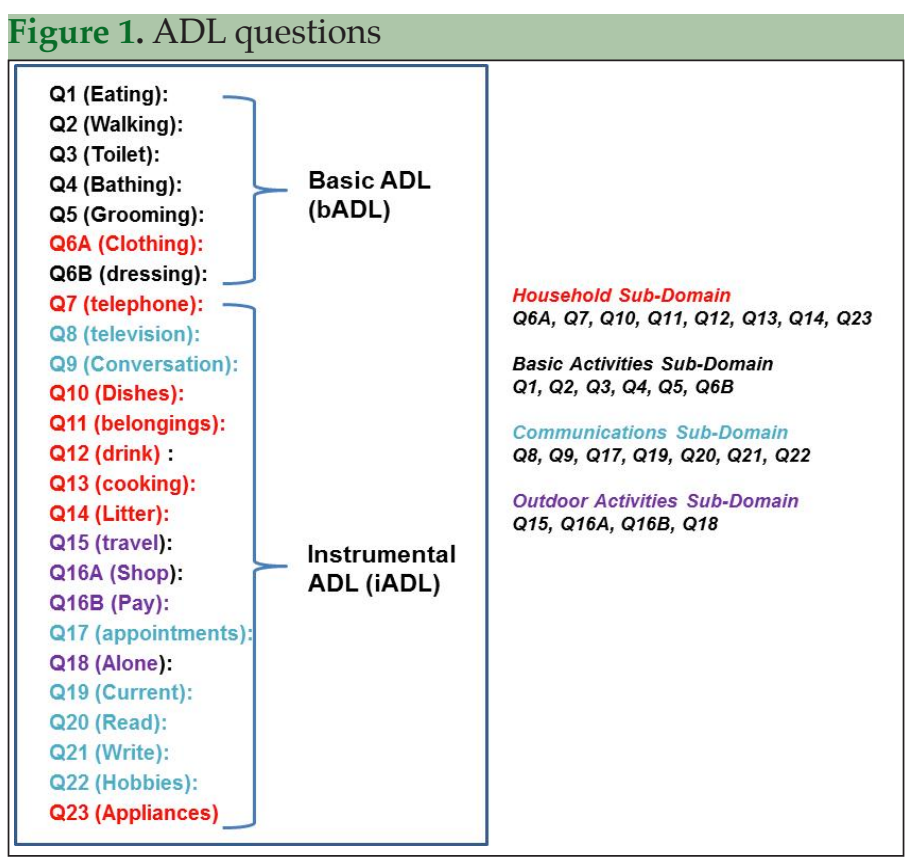

As the assigned level of dependence increased (e.g., Level 1 to Level 5), the associated clinical indicators demonstrated a pattern of greater cognitive impairment (MMSE scores 23.2 to 10.0, $\mathrm{p}<0.001$ ), higher levels of neuropsychiatric disturbance (NPI 3.7 to 27.6; $\mathrm{p}<0.001$ ), 
Table 3. Summary of patient and caregiver characteristics

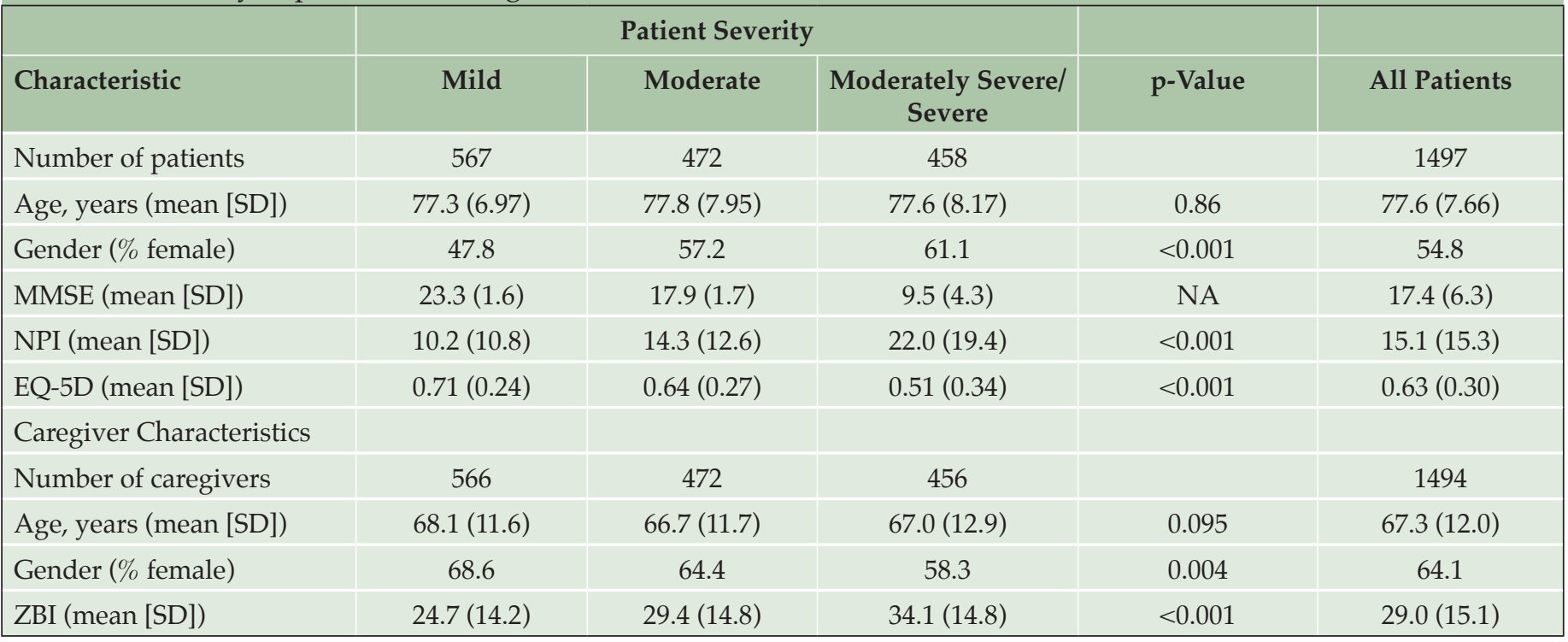

Abbreviations: EQ-5D, EuroQol-5 Dimension; MMSE, Mini-Mental State Examination; NPI12, Neuropsychiatric Inventory-12; SD, standard deviation; ZBI, Zarit Burden Interview; For MMSE and EQ-5D, lower scores indicate greater severity. For NPI and ZBI, higher scores indicate greater severity

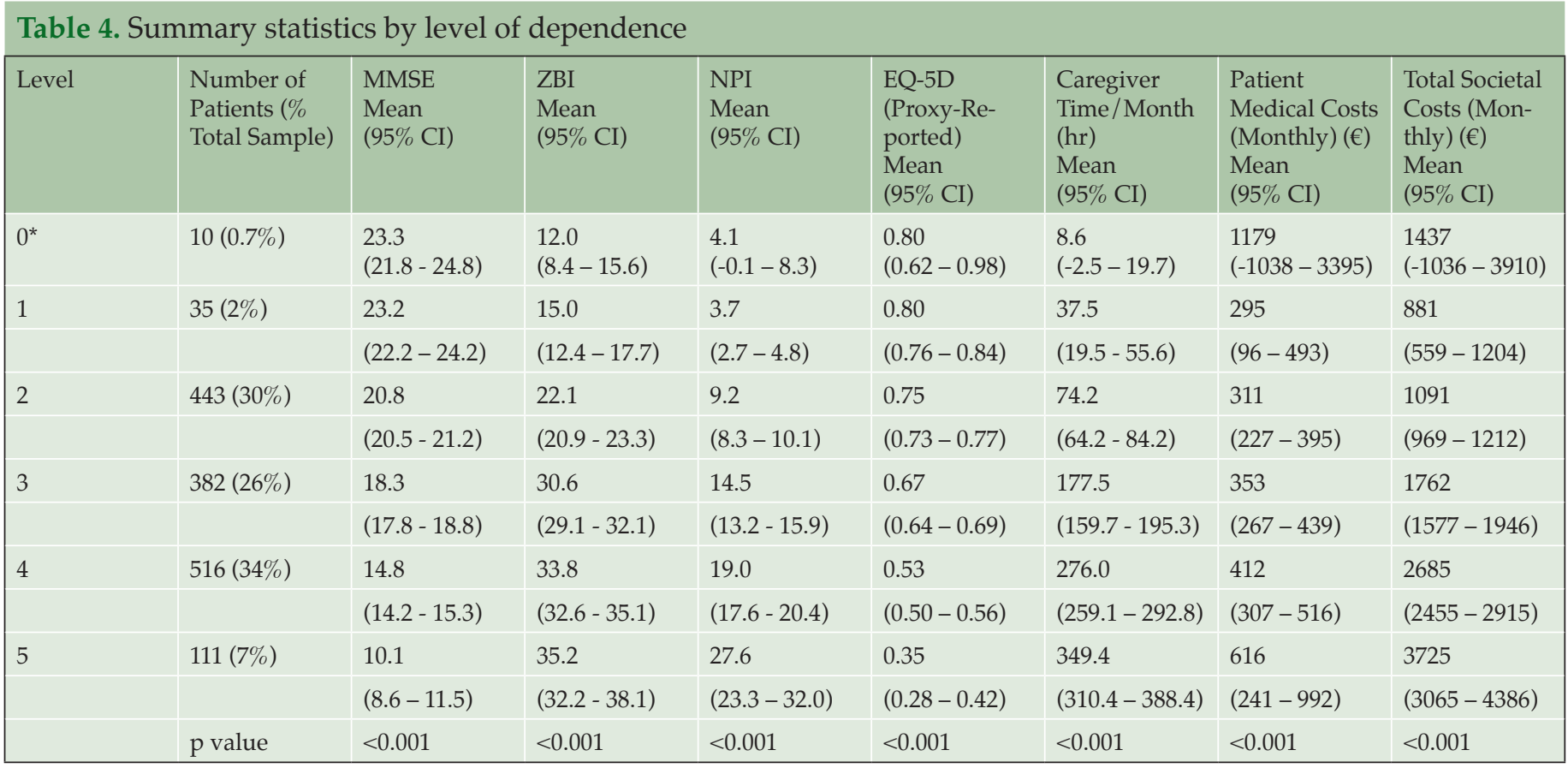

Abbreviations: CI, confidence interval; EQ-5D, EuroQol-5 Dimension; MMSE, Mini-Mental State Examination; NPI12, Neuropsychiatric Inventory-12; ZBI, Zarit Burden Interview; *Based on small sample size.

greater caregiver burden (ZBI 15.0 to 35.2; $\mathrm{p}<0.001$ ), lower quality of life (Patient EQ-5D 0.80 to 0.35; $<<0.001$; Caregiver EQ-5D 0.90 to 0.76; $\mathrm{p}<0.001)$, higher patient direct medical costs per month $(€ 295$ to $€ 616$; $p<0.001)$ and total societal costs per month (€881 to $€ 3725$; $\mathrm{p}<0.001$ ), and caregiver time per month (38 to 349 hours; $\mathrm{p}<0.001$; Table 4).

\section{Discussion}

This initial mapping of ADCS-ADL items onto levels of dependence derived from the DS definitions supports the feasibility of a shared metric for characterizing the functional deficits associated with AD. As the level of dependence increased, other clinical characteristics progressed in an expected manner as cognition worsened, quality of life decreased, and level of neuropsychiatric disturbance, caregiver time, and costs of care all increased. This suggests that levels of dependence are informative and describe intermediate steps along the continuum of AD.

These results are consistent with previous studies that have used the DS itself. Lenderking et al. (2013) (13) measured the internal consistency, validity and 
responsiveness in patients with mild -to -moderately severe AD. They found the DS to be a reliable, valid, and interpretable measure in these patients. Zhu et al. (2008a) (14) estimated the effects of patients' dependence and function on costs of care during the early stages of $\mathrm{AD}$ and found that the DS was associated with higher total cost. A one-point increase in the DS was associated with a $\$ 1,832$ increase in total cost and a $\$ 1,690$ increase in informal cost. A subsequent study by Zhu et al. (2008b) (15) found a one-point increase in the DS score was associated with a $4.1 \%$ increase in caregiving time. The current findings suggest that higher dependence levels may also be associated with increased cost.

Figure 2. Percent of patients in severity category (by MMSE) assigned to each level of dependence

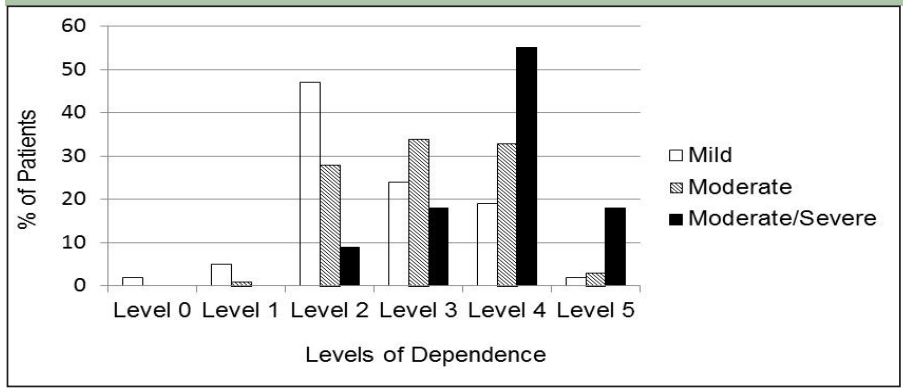

Despite the even stratification of patients by their level of cognitive status (MMSE of mild, moderate, or moderately severe/severe) as specified in the GERAS study design, this same pattern of distribution was not noted for the levels of dependence. Relatively few patients were assigned to Levels 0,1 , or 5 , reflecting the exclusion criteria for GERAS that would preclude enrollment of MCI patients or the most severe patients. The levels of interest for further exploration are therefore Levels 2, 3, and 4. Interestingly, Level 4 included approximately half of the GERAS participants. Although this may indicate a lack of granularity of this scaling scheme when patients reach a moderate level of cognitive impairment, it could also suggest that around this stage of disease, patients' attempts to carry out basic ADL or household activities may not be as successful, and these attempts require more substantial supervision or assistance.

The application of this approach may be particularly useful in the characterization of patients in the mild stage of $\mathrm{AD}$, where discrete clinical milestones are lacking. A majority of GERAS participants in the mild severity category, defined by baseline MMSE, were assigned to a dependence level marked by the need for supervision in completing multiple IADLs (Level 2). The magnitude of change reflected in the clinical and economic characteristics associated with the derived levels of dependence suggests that a transition between levels may represent clinically meaningful disease progression. For example, the burden associated with providing care for a patient with $\mathrm{AD}$ at a Level 3 was $38.5 \%$ greater than the level of burden experienced for caregivers of patients at
Level 2. Likewise, caregiver time spent supervising and assisting with ADLs was 2.39 times greater in Level 3 than Level 2, with significant changes in health utility and total societal costs also observed between these levels. Future efforts to evaluate the impact of treatments on interim milestones of dependence may provide important insights on the value of an intervention to a wide variety of stakeholders.

These functional categories may represent suitable clinical milestones for consideration as endpoints in trials of shorter duration that do not capture the entire spectrum of disease, though further research is needed to understand the timing of patients moving through these stages. Further analyses using longitudinal datasets might look at the number of patients shifting between levels of care over different time frames, with particular attention to patterns of shifts made over the course of 18 months. It is also important to note that the study sample did not cover the entire spectrum of functional impairment. Very mild/asymptomatic and very severe patients were not part of the inclusion criteria. Assessing performance of these dependence levels across the full disease course is thus not possible.

These functional categories might also be applied to other commonly used functional scales using the theoretical model in Table 1. This would require evidence that the application of the theoretical model results in similar staging distributions even across different functional scales. This would have the advantage of creating a shared rubric for classifying dependence level across these different scales.

\section{Funding: This study was supported by Eli Lilly and Company.}

Conflict of interest disclosure: Drs. Wrobleski, Andrews, and Belger are employees of Eli Lilly and Company and / or one of its wholly owned subsidiaries; Drs. Gauthier, Stern, Rentz, and Galasko have received financial compensation from Eli Lilly as scientific consultants.

Ethical standards : «Informed consent was obtained from all participants prior to their enrollment in the study. The study protocol and the informed consent document were reviewed and approved by the Institutional Review Boards at all study sites. The study was conducted in accordance with the ethical principles outlined in the Helsinki Declaration of 1975, as revised in 2000.»

\section{References}

1. Zhu CW, Leibman $C$, Townsend $R$, et al. Bridging from clinical endpoints to estimates of treatment value for external decision makers. J Nutr Health Aging 2009;13:256-259.

2. Stern Y, Albert SM, Sano M, et al. Assessing patient dependence in Alzheimer's disease. J Gerontol 1994;49:M216-222.

3. Jones RW, Romeo R, Trigg R, et al. (2014). Dependence in Alzheimer's disease and service use costs, quality of life, and caregiver burden: The DADE study. Alzheimers Dement, in press.

4. Wimo A, Reed CC, Dodel R, et al. The GERAS study: a prospective observational study of costs and resource use in community dwellers with Alzheimer's disease in three European countries - study design and baseline findings. J Alzheimers Dis 2013;36:385-399.

5. McKhann G, Drachman D, Folstein M, Katzman R, Price D, Stadlan EM. Clinical diagnosis of Alzheimer's disease: report of the NINCDS-ADRDA Work Group under the auspices of Department of Health and Human Services Task Force on Alzheimer's Disease. Neurology 1984;34:939-944.

6. Folstein MF, Folstein SE, McHugh PR. «Mini-mental state». A practical method for grading the cognitive state of patients for the clinician. J Psychiatr Res 1975;12:189-198.

7. Cummings JL. The Neuropsychiatric Inventory: assessing psychopathology 
in dementia patients. Neurology 1997;48(Suppl 6):S10-S16.

8. Wimo A, Jonsson L, Zbrozek A. The resource utilization in Dementia (RUD) instrument is valid for assessing informal care time in community-living patients with dementia. J Nutr Health Aging 2010;14:685-690.

9. The EuroQol Group. Euro-QoL - a new facility for the measurement of health-related quality of life. Health Policy 1990;16:199-208.

10. Zarit SH, Reever KE, Bach-Peterson J. Relatives of the impaired elderly: correlates of feelings of burden. Gerontologist 1980;20:649-655.

11. National Institute of Health and Clinical Excellence. NICE technology appraisal guideline 217. Donepezil, galantamine, rivastigmine and memantine for the treatment of Alzheimer's disease. http://www.nice.org. uk/guidance/TA217. Accessed 19 November 2012.

12. Kahle-Wrobleski K, Coley N, Lepage B, et al. Understanding the complexities of functional ability in Alzheimer's disease: more than just basic and instrumental factors. Curr Alzheimer's Res 2014;11:357-366.

13. Lenderking WR, Wyrwich KW, Stolar M, et al. Reliability, validity, and interpretation of the Dependence Scale in mild to moderately severe Alzheimer's disease. Am J Alzheimer's Dis Other Demen, 2013;28:738-749.

14. Zhu CW, Leibman C, McLaughlin T. The effects of patient function and dependence on costs of care in Alzheimer's disease. J Am Geriatr Soc 2008a;56:1497-1503.

15. Zhu CW, Leibman $C$, McLaughlin $T$, et al. Patient dependence and longitudinal changes in costs of care in Alzheimer's disease. Dement Geriatr Cogn Disord 2008b;26:416-423. 